\title{
An Efficient Encoding for Simplified Protein Structure Prediction Using Genetic Algorithms
}

\author{
Swakkhar Shatabda*†‡, M.A.Hakim Newton* ${ }^{* \ddagger}$, Mahmood A Rashid ${ }^{* \dagger \ddagger}$ and Abdul Sattar* ${ }^{* \dagger}$ \\ *Institute for Integrated and Intelligent Systems (IIIS), Griffith University, Australia \\ †Queensland Research Laboratory (QRL), National ICT of Australia \\ ‡Email: \{s.shatabda,hakim.newton,m.rashid,a.sattar\}@griffith.edu.au
}

\begin{abstract}
Protein structure prediction is one of the most challenging problems in computational biology and remains unsolved for many decades. In a simplified version of the problem, the task is to find a self-avoiding walk with the minimum free energy assuming a discrete lattice and a given energy matrix. Genetic algorithms currently produce the state-of-theart results for simplified protein structure prediction. However, performance of the genetic algorithms largely depends on the encodings they use in representing protein structures and the twin removal technique they use in eliminating duplicate solutions from the current population. In this paper, we present a new efficient encoding for protein structures. Our encoding is nonisomorphic in nature and results into efficient twin removal. This helps the search algorithm diversify and explore a larger area of the search space. In addition to this, we also propose an approximate matching scheme for removing near-similar solutions from the population. Our encoding algorithm is generic and applicable to any lattice type. On the standard benchmark proteins, our techniques significantly improve the state-of-the-art genetic algorithm for hydrophobic-polar (HP) energy model on face-centered-cubic (FCC) lattice.
\end{abstract}

Keywords-Genetic Algorithm; Encoding; Protein Structure Prediction; Simplified Models; HP Model; Lattice;

\section{INTRODUCTION}

Protein structure prediction is one of the most challenging problems in computational biology and remains unsolved for many decades. In a simplified version of the problem, the task is to find a self-avoiding walk with the minimum free energy assuming a discrete lattice and a contact-based energy matrix are given. Genetic algorithms have been successfully used in protein structure prediction and currently produce the state-of-the-art results for the simplified versions. Genetic algorithms [1] are inspired from natural evolution. Population based search algorithms like genetic algorithms depend largely on the success of crossover and mutation over the generations. Diversity in the population of a genetic algorithm is a key issue to address. It has been observed that after a number of generations, diversity is largely affected by the presence of similar or near-similar individuals in the population. Twin removal [2] offers a solution to the problem by removing similar individuals from the population whenever the search gets stuck in a local minima. However, this scheme was incorporated in [3] to remove twins in each generation of the population while generating the individuals. Its important to note that such removal requires a matching scheme.

To find similar individuals in a generation, a representation method is required. In the context of protein structure prediction, the co-ordinates of the amino-acid monomers represent protein structures. However, translational effects in the coordinates make it impossible to match two such structures and hence twin removal is not effective. Absolute encoding that uses the absolute vectors between the three-dimensional points are found in the literature quite abundantly [4]-[7]. A problem with the absolute encoding is that it can not handle the rotational symmetries. Relative encoding [8] is able to handle the problem of rotational symmetry and is used in [3]. This relative encoding requires expensive matrix multiplications each time an absolute vector is transformed into a relative vector. In this paper, we present a new efficient encoding algorithm that represents the vectors as absolute encoding, and handles rotational and transformational symmetry during similarity matching. Our encoding is non-isomorphic and generic to any type of lattice and effective in twin removal and creating necessary diversity. Moreover, we propose an approximate matching scheme for the individuals, where an individual is discarded or removed from the population of a genetic algorithm even if the similarity is not $100 \%$. We apply our techniques within the genetic algorithm framework $\mathrm{GA}^{+}$ [3]. In face centered cubic (FCC) lattice and hydrophobic-polar (HP) energy model, our techniques significantly improve over the existing twin removal methods and improve over the stateof-the-art results on standard benchmark proteins.

The rest of the paper is organized as follows: Section II describes the protein structure prediction problem; Section III reviews the related work; Section IV describes the genetic algorithm framework used; Section V reviews existing protein structure encoding schemes; Section VI describes our approach in detail; Section VII discusses and analyzes the experimental results; and finally, Section VIII presents our conclusions and outlines our future work.

\section{Protein Structure Prediction}

Proteins are involved in almost every process within a living cell including including carrying oxygen (by hemoglobin), signaling cells (by insulin), fighting infection (by antibodies) and performing metabolism (by enzymes). Proper functioning of a protein depdends on the three dimensional native structure that it folds into. Mis-folded proteins are often cause of critical diseases like Alzheimer's disease, Cystic fibrosis, and Mad Cow disease. Knowledge about the native structures of the proteins can lead to understanding of the basics of life and discovery of rational drugs for the related diseases. Since Anfinsen's famous thermodynamic hypothesis [9], it has been believed that every protein has a unique and stable native three dimensional (3D) structure. However, 
due to complex and dynamic nature of the folding process, and the unknown contributing factors of the energy function, protein structure prediction (PSP) has been one of the most challenging problems in biology. In vitro laboratory methods like X-ray crystallography and Nuclear Magnetic Resonance (NMR) spectroscopy are much slow and expensive [10]. These issues have attracted researchers from other fields [11]-[14] to approach the problem using their own techniques.

In absence of any known templates for the proteins, computational methods like homology modeling and protein threading are not applicable. $A b$ initio methods start from scratch and perform a search on the conformational space of structures. High resolution models require all atomic details and are not computationally preferable. Moreover, the contributing factors of different forces of the energy function are unknown and the space of the conformations is very large and complex. Simplified models, though lack many details, provide realistic backbone for the proteins. The candidate structures produced using simplified models could be refined later by adding side chains to achieve the real structures [15].

Proteins are polymers or sequences of amino-acid monomers. In a simplified model, all monomers have an equal size and all bonds are of an equal length. Each amino acid monomer is represented by a single point and its position is restricted to a three dimensional lattice. A simplified energy function is used to calculate the energy of a conformation. The given amino acid sequence fits into a fixed lattice, where every two consecutive monomers in the sequence are also neighbor on the lattice (called the chain constraint) and two monomers can not occupy the same lattice point (called the self avoiding constraint)

\section{A. Lattice}

A lattice $\mathbb{L}$ is a discretization of the continuum of real space where all lattice points are generated by some basis vectors $\vec{v}_{1}, \cdots \vec{v}_{k}$. Two lattice points $p, q \in \mathbb{L}$ are said to be in contact or neighbors of each other, if $q=p+\vec{v}_{i}$ for some vector $\vec{v}_{i}$ in the basis of $\mathbb{L}$. Protein conformations are represented by a sequence of basis vectors (absolute encodings [4]), or by the relative vectors that are relative to the previous basis vectors in the sequence (relative encodings [8]). Different lattices have been considered in the literature (for example, see Fig. 1). The Face Centered Cubic (FCC) lattice is preferred to cubic lattice due to the parity problem [16]. Moreover, the FCC lattice provides (i) the densest packing [17] for spheres of equal size, and (i) the highest degree of freedom for placing an amino acid monomer. Thus, it can provide a realistic discrete mapping for proteins. The FCC lattice is generated by the following basis vectors: $\overrightarrow{v_{1}}=(1,1,0), \overrightarrow{v_{2}}=(-1,-1,0)$, $\overrightarrow{v_{3}}=(-1,1,0), \overrightarrow{v_{4}}=(1,-1,0), \overrightarrow{v_{5}}=(0,1,1), \overrightarrow{v_{6}}=$ $(0,1,-1), \overrightarrow{v_{7}}=(0,-1,-1), \overrightarrow{v_{8}}=(0,-1,1), \overrightarrow{v_{9}}=(1,0,1)$, $\overrightarrow{v_{10}}=(-1,0,1), \overrightarrow{v_{11}}=(1,0,-1), \overrightarrow{v_{12}}=(-1,0,-1)$. The co-ordination number of the FCC lattice is 12 ; which means a point in the lattice has twelve neighbors. Tha basis vectors for cubic lattice are: $\overrightarrow{v_{1}}=(1,0,0), \overrightarrow{v_{2}}=(-1,0,0), \overrightarrow{v_{3}}=(0,1,0)$, $\overrightarrow{v_{4}}=(0,-1,0), \overrightarrow{v_{5}}=(0,0,1), \overrightarrow{v_{6}}=(0,0,-1)$ and coordination number is 6 .

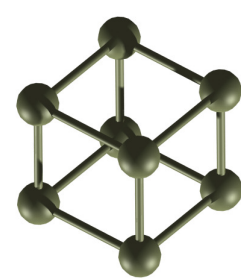

(a)

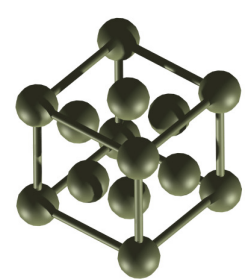

(b)
Figure 1: Different 3D lattices: (a) cubic lattice, and (b) fcc lattice

\section{B. Energy Model}

Lau and Dill [11] proposed a simplified HydrophobicPolar (HP) energy model. In this model, all the amino acids are divided into two groups: hydrophobic H (Gly, Ala, Pro, Val, Leu, Ile, Met, Phe, Tyr, Trp); and hydrophilic or polar P (Ser, Thr, Cys, Asn, Gln, Lys, His, Arg, Asp, Glu). The given amino acid sequence of a protein is represented as a string $s$ of the alphabet $\{H, P\}$. The free energy calculation for the HP model, shown in (1) counts only the energy interactions between two non-consecutive amino acid monomers.

$$
E=\sum_{i, j: i+1<j} c_{i j} \cdot e_{i j}
$$

Here, $c_{i j}=1$ only if two monomers $i$ and $j$ are neighbors (or in contact) on the lattice and 0 otherwise. The other term, $e_{i j}$ is calculated depending on the type of amino acids: $e_{i j}=-1$ if $s_{i}=s_{j}=H$ and 0 otherwise. Minimizing the summation in (1) is equivalent to maximizing the number of non-consecutive $\mathrm{H}-\mathrm{H}$ contacts. HP energy matrix is shown in Table I. There are other simplified energy models in the literature, e.g. variants of HP-model [18], and MJ matrix model [19].

Using the HP energy model together with the FCC lattice, the simplified PSP problem is defined as: given a sequence $s$ of length $n$, find a self avoiding walk $p_{1} \cdots p_{n}$ on the lattice that minimizes the energy defined by (1).

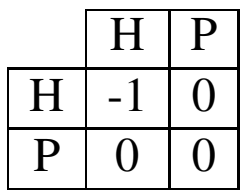

Table I: HP energy model [11]

\section{RELATED WORK}

Protein structure prediction is a hard combinatorial problem even in the simplified forms with square lattice [20] and cubic lattice [21]. There have been attempts by the mathematicians to prove the upper bounds of the variants of the problem [14]. Due to the complexity, several techniques and their hybridizations have been applied to solve the problem. Simulated annealing [12], [22] has been applied to solve the problem due to the similarity with the thermodynamic process of protein folding. Genetic algorithms were first applied to solve this 
problem by Unger and Moult [4], [5]. The basic genetic algorithm was subsequently improved by many researchers [6], [7], [23]. Constraint programming and constraint based methods are used by many researchers [24]-[26]. Lesh et al. [27] provided a novel set of transformations called pull moves extendible to any lattice. Both Lesh et al. [27] and Blazewicz et al. [28] implemented tabu search meta-heuristics independent of each other.

Hybrid techniques that combine the power of different strategies provided better results. Ullah et al. [29] combined local search and constraint programming approaches. Jiang et al. [30] combined tabu search strategy (GTS) with genetic algorithms in the two-dimensional HP Model. Cebrian et al. [31] used tabu search to find 3D structures of Harvard instances [32] on FCC lattices for the first time. In their subsequent work, Dotu et al. [33], [34] applied Large Neighborhood Search (LNS) to further optimize the results found in [31]. Shatabda et al. [35] proposed a memory based approach on top of the algorithm proposed by Dotu et al. [34] and improved the results on the FCC lattice and HP energy model. These results were further improved in a subsequent work [36]. Other methods (such as Ant Colony Optimization (ACO) [37], and Extremal Optimization [38]) are also found in the literature. The stateof-the-art genetic algorithm is proposed in [3].

Several encodings and representation of the protein structures are found in the literature of protein structure prediction. Cartesian co-ordinates were used in [31], [33], [34]. Absolute encodings were in use along with genetic algorithms in [4], [5], [39]. Many researchers have also used relative encodings [3], [35]. Two other non-isomorphic encodings are also found to be used [36], [40].

\section{IV. $\mathrm{GA}^{+}$: A Genetic Algorithm Framework}

In this section we give a brief description of the genetic algorithm framework, $\mathrm{GA}^{+}$proposed in [3]. $\mathrm{GA}^{+}$is an enhanced genetic algorithm for simplified PSP problem. It uses HP energy model and FCC lattice. The pseudocode of $\mathrm{GA}^{+}$ is presented in Algorithm 1. It uses an exhaustive generation approach to diversify the search, a hydrophobic core-directed macro move to intensify the search, and a random-walk algorithm to recover from stagnation. Along with exhaustiveness, macro-move and random-walk, the primitive operators that are implemented in $\mathrm{GA}^{+}$are : single-point crossover, rotation mutation, diagonal move, pull moves, and tilt moves. Detail of the methods of this framework can be found in [3].

\section{Existing EnCODIng Methods}

Several methods of encoding and representation are found in the literature of protein structure prediction problem. We give a brief description of those methods below.

1) Cartesian Co-ordinates:: Each amino acid is independently represented as a point in the Cartesian coordinate. This representation is simple, but it is difficult to handle rotational and translational symmetry using this representation. Moreover, primitive operations like mutation and crossovers becomes computationally expensive. However many local search methods use them because of simplicity.

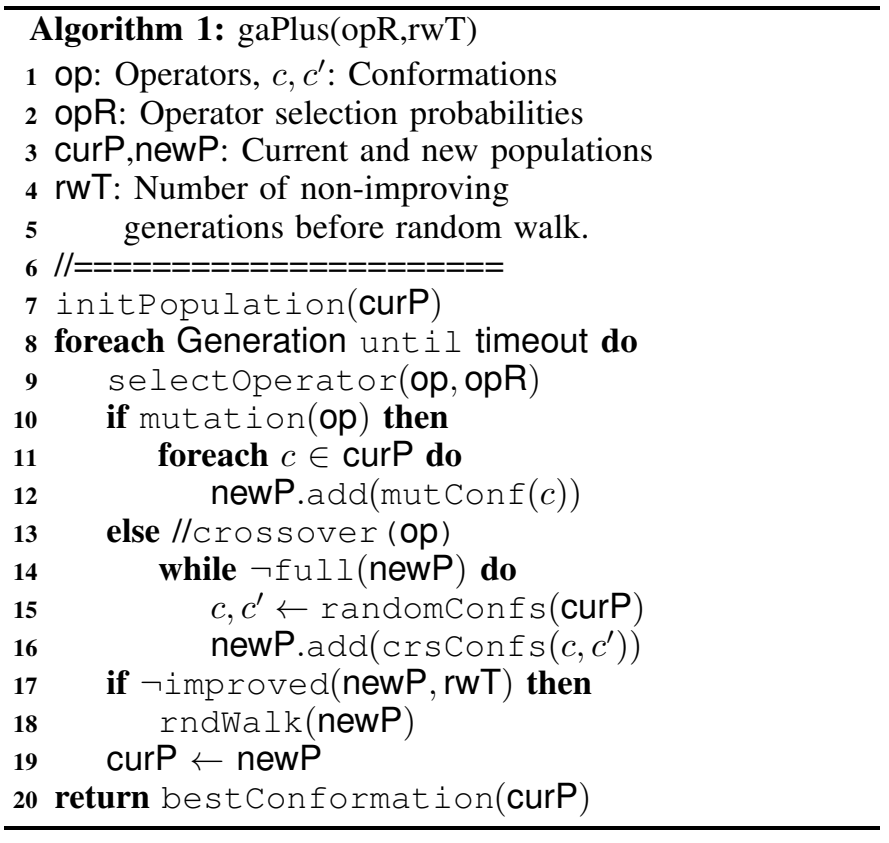

2) Absolute Encoding:: The absolute vectors between coordinates are stored internally to represent protein structures. Local search methods and genetic algorithms use this representation. The encoding and decoding is fast. It assigns a unique symbol for each of the basis vectors needed for a particular type of lattice. For example, in cubic lattice it has six and in FCC lattice it has twelve symbols. However absolute encodings can not handle rotational symmetries. Hence they are not favored when used with twin removal techniques.

3) Relative Encoding:: Relative encoding can solve the rotational symmetry problem of absolute encoding. Rather than directly using the absolute vectors between two coordinate points, in relative encoding every absolute vector is transformed into an absolute vector using the transformation to the base absolute vector. For each of the absolute vectors in a structure are thus transformed into base absolute vectors using matrix multiplication with the updated base matrix. At each step it requires two matrix multiplication operations. This is computationally expensive compared to other encodings but successfully used since it is more efficient in reducing search space [6] and rotational symmetry. Detail of the implementation of relative encoding can be found in [8].

Among other representation torsional angles are used directly in high resolution methods along with Cartesian coordinates. Distance matrices, contact maps and polymer graphs are also found to be used in the literature [41].

\section{OUR METHOD}

In this section, we describe our method of representing the protein structures and the matching scheme for twin removal and diversification by removing approximately similar structures.

\section{A. Representation}

Protein structures are represented using the absolute vectors. There are six different absolute vectors for cubic lattice 
and twelve different vectors for FCC lattice. These vectors are found from the co-ordinates assigned to the amino-acid monomers. Two structures in cubic lattice are given in Figure 2. Corresponding absolute vectors are also given in Table II.
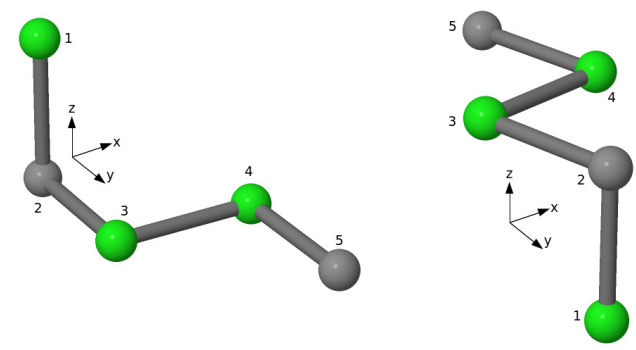

Figure 2: Two identical structures in cubic lattice having different absolute encodings

Encoding and decoding of the structures are very simple. For encoding, we assign symbols for each of the different vectors. While decoding we lookup for the absolute vectors for the symbols and generate the points. Pseudo-code for encode and decode are given in Algorithm 2 and Algorithm 3 respectively.

\begin{tabular}{|c|c|c|c|c|}
\hline Vector ID & 1 & 2 & 3 & 4 \\
\hline Structure 1 & $(0,0,-1)$ & $(0,1,0)$ & $(1,0,0)$ & $(0,1,0)$ \\
\hline Structure 2 & $(0,0,1)$ & $(0,-1,0)$ & $(1,0,0)$ & $(0,-1,0)$ \\
\hline
\end{tabular}

Table II: Absolute vectors for the two structures in Figure 2
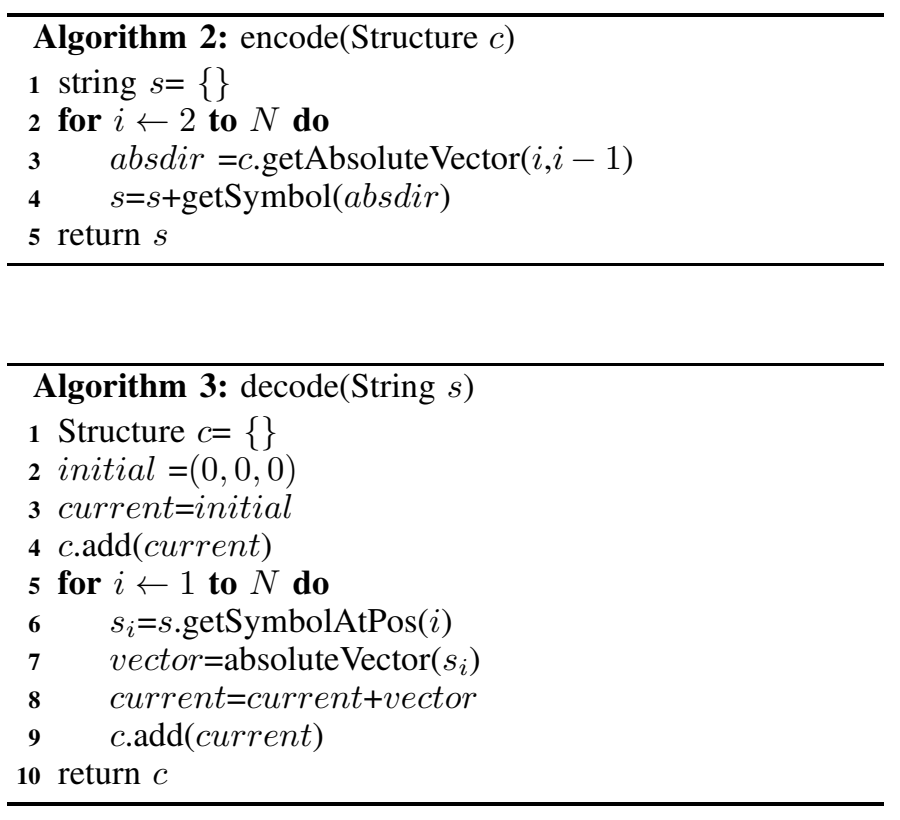

For example, in cubic lattice we can assign six symbols, U $($ up $)=(0,0,1), \mathrm{D}($ down $)=(0,0,-1), \mathrm{F}$ (forward $)=(1,0,0)$, $\mathrm{B}$ (backward) $=(-1,0,0), \mathrm{R}$ (right) $=(0,1,0)$ and $\mathrm{L}$ (left) $=(0,-1,0)$. So when we encode the structures of Figure 2 they become encoded as, "DRFR" and "ULFL" respectively. This encoding is similar to the absolute encoding in literature.
The vectors in FCC lattice are also encoded and decoded in a similar way.

\section{B. Matching}

The matching part for absolute encodings are very simple in literature. They simply match the encoded string using string matching algorithms. However, we notice this is not suitable for effective twin removal. If we compare two encoded structures in Figure 2 using string matching we see that, "DRFR" and "ULFL" are very different from each other. Traditional matching in genetic algorithms can not detect from the encodings that these two structures are actually identical only different by some rotation. These kinds of encoding are called iso-morphic encodings, since they can not detect the rotational symmetry. Relative encodings [8] offer a solution for the problem. But they require expensive matrix multiplication operations each time of decoding or encoding a symbol. Details of the method is given in [8]. However a non-isomorphic encoding for cubic lattices are given in [40] where angle computations are needed and their algorithm does not take into account the different senses of the vectors. They also use string matching for comparison between structures. We propose a novel matching scheme for absolute encodings that ensures the non-isomorphic property and does not require expensive computation.
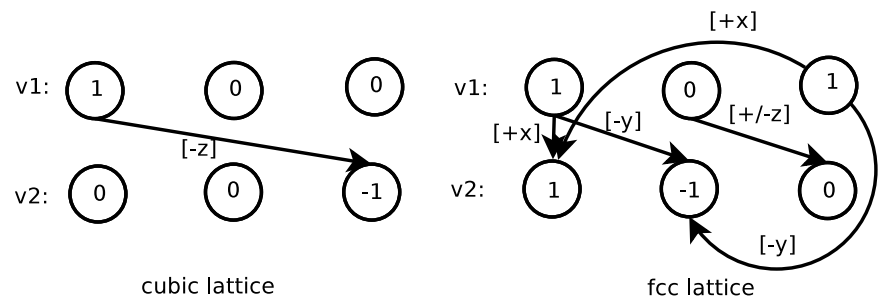

Figure 3: Partial mapping of absolute vectors

Our encoding starts with empty mapping of the vector positions. Whenever we encounter an absolute vector from one structure, we try to match the non-zero positions with the absolute vector of the another structure. The sign is also assigned. In a particular absolute vector, only a few positions are non-zero. For cubic lattices we find only 1 non-zero position. So the partial mapping starts with that position only. The sign changes are also taken into account. In Figure 3 we see that, there is a match between non-zero position $x$ of $v_{1}$ with the non-zero position $z$ of $v_{2}$. Therefore, mapping for this $x$ becomes $-z$ since the sign is reversed. With this partial mapping the partial matching continues. The full map is found as soon as non-zero are found in other positions in consecutive absolute vectors.

In case of FCC lattice there are two non-zero positions. In Figure 3 we find that, there is a match of position $x$ and $z$ of vector $v_{1}$ with the positions $x$ and $y$ from vector $v_{2}$. Since there are two matches there will to two partial maps. We also note that, in case of FCC lattice, the matching position for $y$ is also found since there is only one single zero position in both of the vectors. But the sign for this mapping is not decided yet. Matching with partial map will continue until the full map is found. Pseudo-code for mapping two vectors is given in Algorithm 4. 


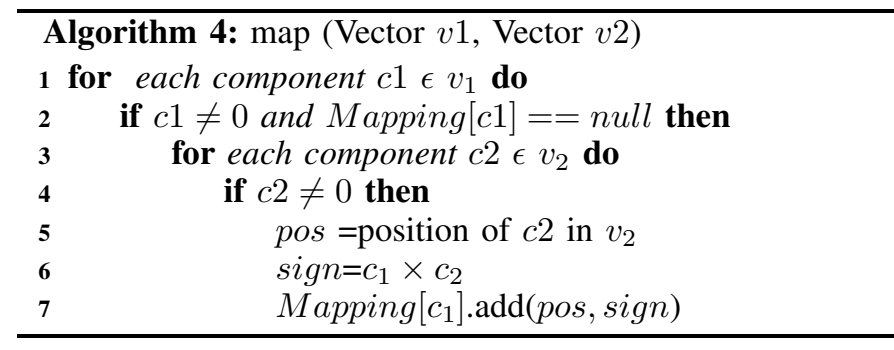

Whenever there is a mismatch in the vectors, a mismatch counter is increased. We say two structures are different and no match was found if this mismatch counter is greater than a certain proximity, $p$. For $100 \%$ similarity matching or twin removal the proximity should be set to zero. The automaton for matching of absolute vectors are given in Figure 4.

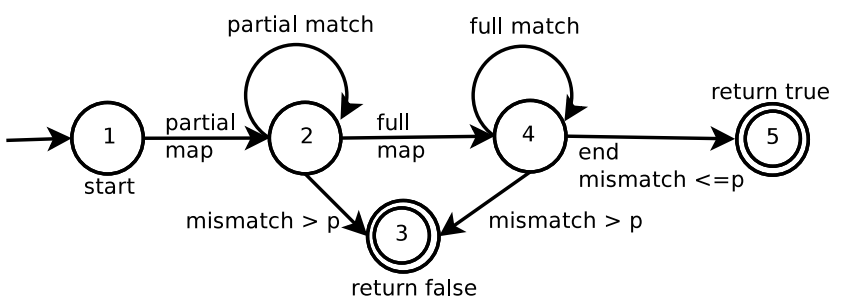

Figure 4: Matching automaton for two structures

Using this matching algorithm it is possible now to detect that the two structures in Figure 2 are identical. In the first vector we find a mapping $[-z]$ for $z$. Then in the second vector, we get mapping $[-y]$ for $y$. In the third vector, we get $[+x]$ for $x$. At this stage we get the full mapping. Now for the fourth vector we get, $(0,1,0)$, using our mapping we get, $(0,-1,0)$ which is identical to the fourth vector of structure 2 . Here the matching ends and and since no mismatch was found, our algorithm will return true. Pseudo-code for matchin is given in Algorithm 5. This matching algorithm runs in $O(n)$ time where $n$ is the length of the protein sequence.

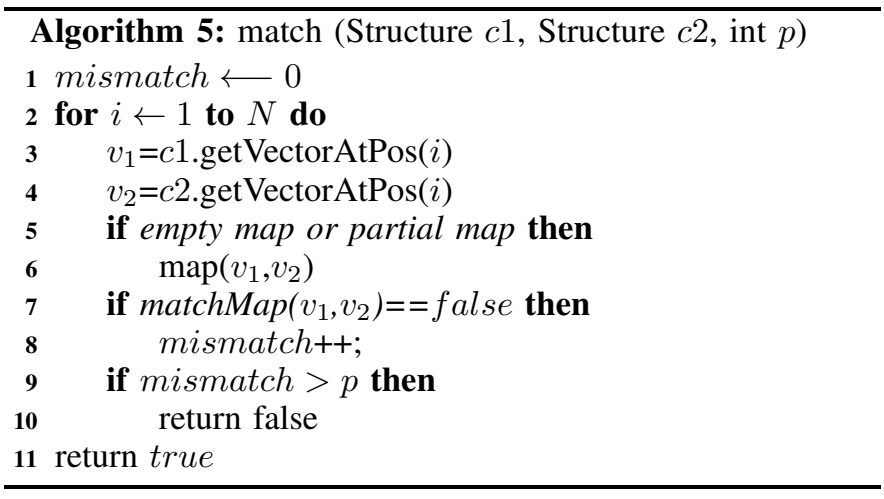

\section{Implementation}

We used the source code of $\mathrm{GA}^{+}$implementation in [3]. The program in written in Java. $\mathrm{GA}^{+}$used relative encoding to represent the structures and string matching for similarity comparison. We altered the encoding, decoding and matching algorithms in $\mathrm{GA}^{+}$. The matching algorithm has a proximity parameter $p$ which is set to the amount of mismatch for a particular percentage of mapping. The effect of this approximate matching is also discussed in Section VII.

\section{EXPERIMENTAL RESULTS}

We ran experiments on the NICTA (www.nicta.com.au) cluster machine with a maximum limit on time. The cluster has a number of machines each equipped with 2 quad-core CPUs (Intel Xeon@2.0GHz,6MB L2 Cache) and 16GB RAM (2GB per core), running Rocks OS (a Linux variant for cluster). For each of the protein sequences we performed 20 runs and each run was set to finish after 5 hours. We incorporated our encoding and approximate matching scheme within the $\mathrm{GA}^{+}$ framework [3]. We kept all other parameters of the algorithm the same except encoding, decoding and matching scheme. Three sets of results are presented in Table III with three different encodings: absolute encoding, relative encoding and our encoding. Note that, the results with relative encoding represents previous state-of-the-art results. We have selected two sets of benchmarks for our experiments.

\section{A. Benchmark Set-I}

The first benchmark set that we used is taken from Sebastian Will's PhD thesis [42] and publicly available in the website of Peter Clote Laboratory ${ }^{1}$. These are the $R$ sequences and $F 180$ sequences of length 200 and 180 respectively. The best and average energy levels achieved are reported in the upper part of Table III. Optimal lower bounds of native energy level for each of the proteins are also reported in the column ' $E_{l}$ '.

\section{B. Benchmark Set-II}

We also use a second set of benchmarks originally used in [35] which are derived from the famous Critical Assessment of Techniques for Protein Structure Prediction (CASP) competition $^{2}$. Six proteins were randomly chosen from the target list that are around the length of $230 \pm 50$. These sequences were then converted to HP sequences depending on the nature of the amino-acids in the sequences. Results for these six proteins are also reported in Table III (lower part). The PDB id for each of these proteins is also given and the parameter settings for these six proteins were kept the same.

\section{Result Analysis}

The bold faced values in Table III are the best values found for each of the proteins. It is clearly evident that for all the proteins our algorithm achieves average energy levels no worse than other encodings and hence improves over the state-of-theart results. Moreover, in most of the cases our algorithm is able to find best solutions compared to other encodings.

\section{Relative Improvement}

We report the relative achievement of our approach measured in terms of the difference with optimal bound of the energy level in the "RI" column of Table III. This value is significant because it gets harder to find better conformations as the energy level of a protein sequence approaches the

\footnotetext{
${ }^{1}$ http://bioinformatics.bc.edu/clotelab/FCCproteinStructure/

${ }^{2} \mathrm{http}: / /$ predictioncenter.org/casp9/targetlist.cgi
} 


\begin{tabular}{|c|c|c|c|c|c|c|c|c|c|c|c|}
\hline \multicolumn{4}{|c|}{ Protein Info } & \multicolumn{2}{|c|}{ Our Encoding } & \multicolumn{3}{|c|}{ Absolute Encoding } & \multicolumn{3}{|c|}{ Relative Encoding [8] } \\
\hline No & id & size & $E_{l}$ & best & avg & best & avg & RI & best & avg & RI \\
\hline 1 & F180_1 & & -378 & -355 & -346 & -347 & -337 & $21.95 \%$ & -350 & -340 & $15.78 \%$ \\
\hline 2 & F180_2 & 180 & -381 & -362 & -350 & -352 & -343 & $18.42 \%$ & -351 & -343 & $18.42 \%$ \\
\hline 3 & F180_3 & & -378 & -366 & -359 & -360 & -351 & $29.62 \%$ & -367 & -352 & $26.92 \%$ \\
\hline 4 & $\mathrm{R} 1$ & & -384 & -359 & -347 & -360 & -343 & $9.75 \%$ & -355 & -345 & $5.12 \%$ \\
\hline 5 & $\mathrm{R} 2$ & 200 & -383 & -364 & -353 & -357 & -344 & $23.07 \%$ & -353 & -342 & $26.82 \%$ \\
\hline 6 & $\mathrm{R} 3$ & & -385 & -363 & -349 & -360 & -343 & $14.28 \%$ & -364 & -343 & $14.28 \%$ \\
\hline 7 & $3 \mathrm{mse}$ & 179 & -323 & -292 & -284 & -283 & -277 & $15.21 \%$ & -287 & -279 & $11.36 \%$ \\
\hline 8 & $3 \mathrm{mr} 7$ & 189 & -355 & -329 & -320 & -319 & -312 & $18.60 \%$ & -329 & -320 & $0 \%$ \\
\hline 9 & $3 \mathrm{mqz}$ & 215 & -474 & -422 & -407 & -417 & -404 & $4.28 \%$ & -427 & -407 & $0 \%$ \\
\hline 10 & 3no6 & 229 & -455 & -413 & -403 & -414 & -395 & $13.33 \%$ & -407 & -397 & $10.34 \%$ \\
\hline 11 & 3 no3 & 258 & -494 & -421 & -400 & -421 & -396 & $4.08 \%$ & -413 & -398 & $2.08 \%$ \\
\hline 12 & 3no7 & 279 & $\mathrm{n} / \mathrm{a}$ & -497 & -484 & -505 & -484 & $\mathrm{n} / \mathrm{a}$ & -511 & -483 & $\mathrm{n} / \mathrm{a}$ \\
\hline
\end{tabular}

Table III: Experimental results of $\mathrm{GA}^{+}$with our encoding, absolute encoding and relative encoding [8].

\begin{tabular}{|c|ccc|ccc|ccc|}
\hline \multirow{2}{*}{$\begin{array}{c}\text { Protein } \\
\text { ID }\end{array}$} & \multicolumn{3}{|c|}{ Our Encoding } & \multicolumn{3}{c|}{ Absolute Encoding } & \multicolumn{3}{c|}{ Relative Encoding [8] } \\
\cline { 2 - 10 } & solutions & twins & $\%$ & solutions & twins & $\%$ & solutions & twins & $\%$ \\
\hline F180_1 & 35.13 & 1.2231 & $\mathbf{3 . 4 8}$ & 25.15 & 0.78142 & 3.1 & 30.79 & 0.85799 & 2.7 \\
F180_2 & 32.576 & 1.0715 & $\mathbf{3 . 2 9}$ & 36.90 & 1.2566 & $3.4^{*}$ & 30.58 & 0.8599 & 2.8 \\
F180_3 & 34.607 & 1.1574 & $\mathbf{3 . 3 4}$ & 30.969 & .9926 & 3.2 & 29.30 & 0.67669 & 2.31 \\
\hline R1 & 27.79 & 0.74654 & $\mathbf{2 . 6 8}$ & 31.231 & 0.80676 & 2.58 & 24.28 & 0.42685 & 1.75 \\
R2 & 27.94 & 0.68009 & $\mathbf{2 . 4 3}$ & 25.790 & 0.6342 & $2.45^{*}$ & 21.16 & 0.32785 & 1.54 \\
R3 & 29.77 & 0.7177 & $\mathbf{2 . 4 1}$ & 24.816 & 0.62293 & $2.51^{*}$ & 26.66 & 0.6377 & 2.39 \\
\hline 3mse & 32.19 & 1.0563 & $\mathbf{3 . 2 8}$ & 37.449 & 0.13533 & 0.36 & 36.235 & 1.1183 & 3.08 \\
3mr7 & 30.04 & 0.84448 & $\mathbf{2 . 9}$ & 24.114 & 0.29868 & 1.23 & 25.51 & 0.46501 & 1.82 \\
3mqz & 23.647 & 4.3276 & $\mathbf{1 . 8 3}$ & 25.002 & 0.50921 & $2.03^{*}$ & 21.1 & 0.39089 & 1.85 \\
\hline 3no6 & 24.15 & 0.46891 & $\mathbf{1 . 9 4}$ & 23.501 & 0.42692 & 1.81 & 21.736 & 0.30922 & 1.42 \\
3no3 & 18.06 & 0.27225 & $\mathbf{1 . 5 1}$ & 18.248 & 0.29276 & $1.60^{*}$ & 19.64734 & 0.25047 & 1.27 \\
3no7 & 12.79 & 0.15949 & $\mathbf{1 . 2 4}$ & 17.421 & 0.25864 & $1.48^{*}$ & 12.362 & 0.13055 & 1.05 \\
\hline
\end{tabular}

Table IV: Average number of solutions generated (in millions) and twins discarded (in millions) by our encoding, absolute encoding and relative encoding [8], each protein was given 20 runs, each with 5 hours time limit.

optimal. Similar measurements are also used in [35]. Relative improvement (R.I.) is defined as below:

$$
\text { R.I. }=\frac{E_{o}-E_{r}}{E_{l}-E_{r}} \times 100 \%
$$

where $E_{o}$ is the average energy level achieved by our approach, $E_{r}$ is the average energy level achieved by the other approach, and $E_{l}$ is the optimal lower bound of the energy level. The missing values indicate the absence of any lower bound for the corresponding protein sequence. Relative improvement for absolute encodings ranges from $4 \%$ to $29 \%$. Only for one protein 3 no7 the average of energy level achieved are equal. In case of Relative Encoding [8], relative improvement ranges from $2 \%$ to $26 \%$ except that there are two cases where average energy level achieved by both encodings are same. We clearly see that our algorithm produces structures that significantly improves over other encodings and state-of-the-art method in terms of average energy level achieved.

\section{E. Effective Twin Removal}

In Table IV we report the average total number of solutions generated by $\mathrm{GA}^{+}$algorithm with our encoding, absolute encoding and relative encoding [8]. We also report average number of twins discarded and the percentage of that to the total number for each of the encodings. The best percentage values are shown in bold faced fonts. From the values reported in column 'twins' we see that for each of the proteins our encoding discards more twins in number than the relative encoding. Successful twin removal effects in the search process by creating necessary diversification and the improvement in the result is evident for that. Moreover, we observe that for each of the proteins number of solutions generated is larger for our encoding. It is an indication of the efficiency in computation and matching and also shows that $\mathrm{GA}^{+}$traverses a greater search space using our encoding compared to relative encoding, given the same time span. In case of absolute encoding, its faster because of the string matching and supposed to generate and discard more twins. However, it can not solve the problem with rotational symmetry. The asterix marked 


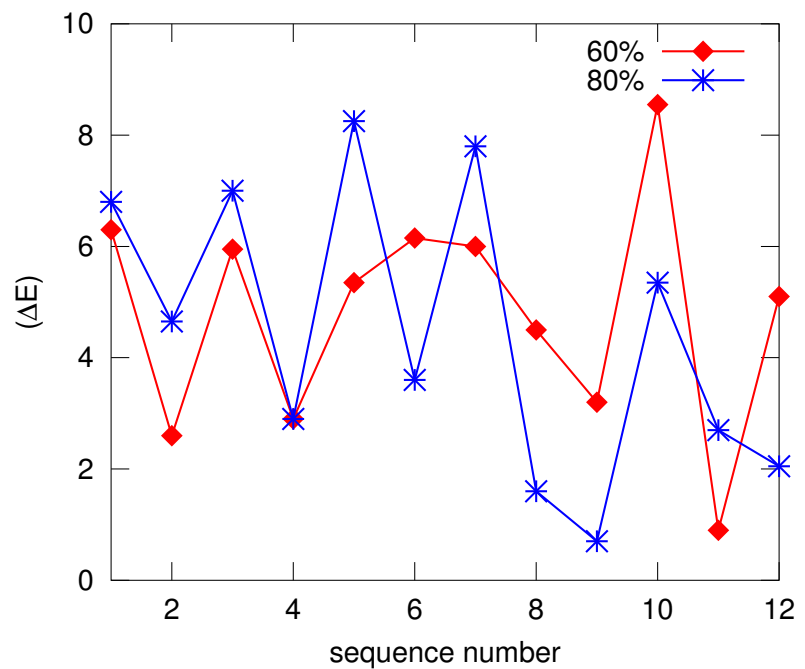

Figure 5: Gain in average energy level $(\Delta E)$ of $60 \%$ and $80 \%$ approximate matching over $100 \%$ matching for different proteins, $\Delta E$ is defined in Eq. 3, where $x \%$ is the approximate percentage of similarity.

values are cases where absolute encoding was able to find more twins in terms of percentage to the total number of solutions generated. However, the twins it discards are only translational isomorphic.

\section{F. Effect of Approximate Matching}

We ran the same experiments by varying our matching proximity measure. In case of twin removal, generally two structures are considered to be same if they are $100 \%$ similar. Using our encoding, we varied this proximity measure by $80 \%$ and $60 \%$. It resulted in an increase in discarded solution which is very natural and also improved the performance of search. In Figure 5, we show the average improvement, $\Delta \mathrm{E}$ of approximate matchings over the $100 \%$ matching for twin removal. In all the proteins the approximate matchings were able to improve over the $100 \%$ matchings.

$$
\Delta E^{x \%}=E n e r g y_{\text {average }}^{x \%}-\text { Energy } y_{\text {average }}^{100 \%}
$$

\section{G. Search Progress}

To observe the effect of our encoding on the search we plot the average energy level achieved by $\mathrm{GA}^{+}$with all three encodings over five hours for protein R1 in Figure 6. Notice that, from the beginning there is a fall in the energy in our technique and it continues to improve, while the behavior of other two encodings are almost similar.

\section{CONCLUSION}

In this paper, we have presented a new efficient encoding for protein structures. Our encoding is non-isomorphic in nature and results in better twin removal. We also propose an approximate matching scheme for twin removal which enables near-similar solutions to be discarded from the population of

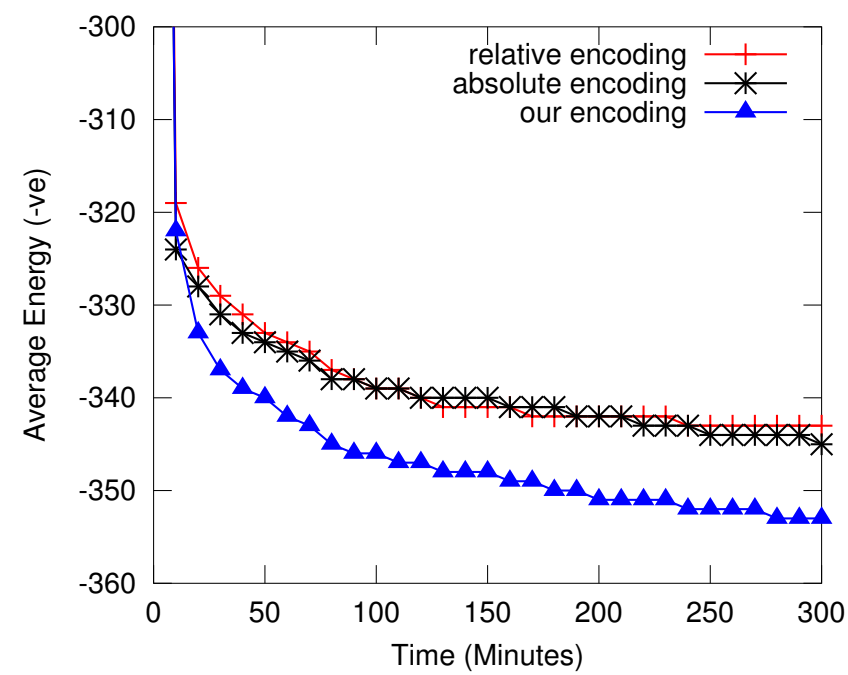

Figure 6: Search progress of $\mathrm{GA}^{+}$for protein $\mathrm{R} 1$ with time for different encodings

genetic algorithms. Our technique is found to improve the state-of-the-art method on standard benchmark proteins. In future, we wish to apply such encodings to other constrained problems where genetic algorithms are applied and search space can be reduced by such matching techniques. We also wish to apply this technique to other local search algorithms and apply memory to genetic algorithms along with the encoding to see the effects on simplified protein structure prediction problem.

\section{ACKNOWLEDGEMENT}

NICTA is funded by the Australian Government as represented by the Department of Broadband, Communications and the Digital Economy and the Australian Research Council through the ICT Centre of Excellence program.

\section{REFERENCES}

[1] J. H. Holland, "Outline for a logical theory of adaptive systems," Journal of the ACM (JACM), vol. 9, no. 3, pp. 297-314, 1962.

[2] M. T. Hoque, M. Chetty, A. Lewis, and A. Sattar, "Twin Removal in Genetic Algorithms for Protein Structure Prediction using Low-Resolution Model," Transactions on Computational Biology and Bioinformatics, vol. 8, no. 1, pp. 234-245, 2011.

[3] M. A. Rashid, M. Hoque, M. Newton, D. Pham, and A. Sattar, "A new genetic algorithm for simplified protein structure prediction," in AI 2012: Advances in Artificial Intelligence, ser. Lecture Notes in Computer Science, 2012.

[4] R. Unger, "The genetic algorithm approach to protein structure prediction," Applications of Evolutionary Computation in Chemistry, pp. 2697-2699, 2004.

[5] R. Unger and J. Moult, "On the applicability of genetic algorithms to protein folding," in System Sciences, 1993, Proceeding of the TwentySixth Hawaii International Conference on, vol. 1. IEEE, 1993, pp. $715-725$

[6] N. Krasnogor, W. E. Hart, J. Smith, and D. A. Pelta, "Protein structure prediction with evolutionary algorithms," in Proceedings of the genetic and evolutionary computation conference, vol. 2. Citeseer, 1999, pp. 1596-1601. 
[7] R. Konig and T. Dandekar, "Improving genetic algorithms for protein folding simulations by systematic crossover." Biosystems, vol. 50, no. 1, pp. 17-25, Apr. 1999. [Online]. Available: http://www.ncbi.nlm.nih.gov/pubmed/10235648

[8] R. Backofen, S. Will, and P. Clote, "Algorithmic approach to quantifying the hydrophobic force contribution in protein folding," in Proceedings of the Pacific Symposium on Biocomputing, 2000, pp. 92-103.

[9] C. B. Anfinsen, "The Principles that Govern the Folding of Protein Chains," Science, vol. 181, no. 4096, pp. 223-230, 1973.

[10] D. Baker and A. Sali, "Protein structure prediction and structural genomics," Science, vol. 294, pp. 93-96, 2001.

[11] K. F. Lau and K. A. Dill, "A lattice statistical mechanics model of the conformational and sequence spaces of proteins," Macromolecules, vol. 22, no. 10, pp. 3986-3997, 1989.

[12] H. Kawai, T. Kikuchi, and Y. Okamoto, "A prediction of tertiary structures of peptide by the Monte Carlo simulated annealing method," Protein Engineering, vol. 3, no. 2, p. 85, 1989.

[13] G. W. Klau, N. Lesh, J. Marks, and M. Mitzenmacher, "Human-Guided Tabu Search," in The Eighteenth National Conference on Artificial Intelligence (AAAI-02), 2002.

[14] W. E. Hart and S. Istrail, "Lattice and off-lattice side chain models of protein folding: linear time structure prediction better than $86 \%$ of optimal," Journal of Computational Biology, vol. 4, no. 3, pp. 241-259, 1997.

[15] P. Rotkiewicz and J. Skolnick, "Fast procedure for reconstruction of full-atom protein models from reduced representations," Journal of Computational Chemistry, vol. 29, no. 9, pp. 1460-1465, 2008.

[16] S. E. Decatur, "Protein folding in the generalized hydrophobic-polar model on the the triangular lattice," Tech. Rep., 1996.

[17] B. Cipra, "Packing challenge mastered at last." Science, vol. 281, no. 5381, p. 1267, 1998.

[18] E. Bornberg-Bauer, "Chain growth algorithms for hp-type lattice proteins," in Proceedings of the first annual international conference on Computational molecular biology, ser. RECOMB '97. New York, NY, USA: ACM, 1997, pp. 47-55.

[19] S. Miyazawa and R. L. Jernigan, "Estimation of effective interresidue contact energies from protein crystal structures: quasi-chemical approximation," Macromolecules, vol. 18, no. 3, pp. 534-552, Mar. 1985.

[20] P. Crescenzi, D. Goldman, C. Papadimitriou, A. Piccolboni, and M. Yannakakis, "On the complexity of protein folding," Journal of Computational Biology, vol. 5, pp. 597-603, 1998.

[21] B. Berger and T. Leighton, "Protein folding in the hydrophobichydrophilic(hp) is np-complete," in Proceedings of the second annual international conference on Computational molecular biology, ser. RECOMB '98, 1998, pp. 30-39.

[22] K. Steinhofel, A. Skaliotis, and A. Albrecht, "Logarithmic Simulated Annealing for Protein Folding," 2007.

[23] M. T. Hoque, M. Chetty, and L. S. Dooley, "A guided genetic algorithm for protein folding prdiction using 3D hyrophobic-hydrophilic model," in IEEE Congress on Evolutionary Computation. IEEE Congress on Evolutionary Computation, 2006, pp. 2339-2346.

[24] K. Yue and K. A. Dill, "Forces of tertiary structural organization in globular proteins," Proceedings of the National Academy of Sciences of the United States of America, vol. 92, no. 1, p. 146, 1995.

[25] M. Mann, S. Will, and R. Backofen, "CPSP-tools - Exact and complete algorithms for high-throughput 3D lattice protein studies," $B M C$ Bioinformatics, vol. 9, no. 1, p. 230, 2008.

[26] D. P. Alessandro, A. Dovier, and E. Pontelli, "A constraint solver for discrete lattices, its parallelization, and application to protein structure prediction," Softw. Pract. Exper, vol. 37, pp. 1405-1449, November 2007.

[27] N. Lesh, M. Mitzenmacher, and S. Whitesides, "A complete and effective move set for simplified protein folding," in Research in comp. mol. biology (RECOMB), 2003.

[28] J. Blazewicz, K. Dill, P. Lukasiak, and M. Milostan, "A Tabu search strategy for finding low energy structures of proteins in HP-model," Computational Methods in Science and Technology, vol. 10, pp. 7-19, 2004.
[29] A. D. Ullah and K. Steinhöfel, "A hybrid approach to protein folding problem integrating constraint programming with local search," $B M C$ bioinformatics, vol. 11, no. Suppl 1, p. S39, 2010.

[30] T. Jiang, Q. Cui, G. Shi, and S. Ma, "Protein folding simulations of the hydrophobic-hydrophilic model by combining tabu search with genetic algorithms," The Journal of chemical physics, vol. 119, p. 4592, 2003.

[31] M. Cebrián, I. Dotú, P. Van Hentenryck, and P. Clote, "Protein structure prediction on the face centered cubic lattice by local search," in Proceedings of the 23rd national conference on Artificial intelligence Volume 1, 2008, pp. 241-246.

[32] K. Yue, K. M. Fiebig, P. D. Thomas, H. S. Chan, E. I. Shakhnovich, and K. A. Dill, "A test of lattice protein folding algorithms," Proceedings of the National Academy of Sciences of the United States of America, vol. 92, no. 1, p. 325, 1995.

[33] I. Dotu, M. Cebrián, P. Van Hentenryck, and P. Clote, "Protein structure prediction with large neighborhood constraint programming search," in Principles and Practice of Constraint Programming. Springer, 2008, pp. 82-96.

[34] I. Dotu, M. Cebrian, P. Van Hentenryck, and P. Clote, "On lattice protein structure prediction revisited," Computational Biology and Bioinformatics, IEEE/ACM Transactions on, vol. 8, no. 6, pp. 1620-1632, nov.-dec. 2011.

[35] S. Shatabda, M. Newton, D. N. Pham, and A. Sattar, "Memory-based local search for simplified protein structure prediction," in ACM Conference on Bioinformatics, Computational Biology and Biomedicine. ACM, 2012, pp. 345-352.

[36] S. Shatabda, M. Hakim Newton, M. Rashid, D. Pham, and A. Sattar, "The road not taken: retreat and diverge in local search for simplified protein structure prediction," BMC Bioinformatics, vol. 14, no. Suppl 2, p. S19, 2013.

[37] A. Shmygelska and H. H. Hoos, "An ant colony optimisation algorithm for the 2D and 3D hydrophobic polar protein folding problem," $B M C$ bioinformatics, vol. 6, no. 1, p. 30, 2005.

[38] H. Lu and G. Yang, "Extremal optimization for protein folding simulations on the lattice," Comput. Math. Appl., vol. 57, pp. 1855-1861, June 2009.

[39] M. Khimasia and P. Coveney, "Protein structure prediction as a hard optimization problem: The genetic algorithm approach," Molecular Simulation, vol. 19, pp. 205-226, 1997.

[40] M. T. Hoque, M. Chetty, and L. S. Dooley, "Non-Isomorphic Coding in Lattice Model and its Impact for Protein Folding Prediction Using Genetic Algorithm," in IEEE Symposium on Computational Intelligence and Bioinformatics and Computational Biology. IEEE Symposium on Computational Intelligence and Bioinformatics and Computational Biology, 2006, pp. 1-8.

[41] K. Fiebig and K. Dill, "Protein core assembly processes," The Journal of chemical physics, vol. 98, p. 3475, 1993.

[42] S. Will, Exact, constraint-based structure prediction in simple protein models. PhD Thesis, 2005. 